\title{
A Physical Space-modeled Approach to Lagrangian Equations with Bundle Structure for Minkowski 4-space
}

\author{
Simge Simsek*, Cansel Yormaz \\ Department of Mathematics, Pamukkale University, Denizli, 20070, Turkey
}

Copyright (C)2017 by authors, all rights reserved. Authors agree that this article remains permanently open access under the terms of the Creative Commons Attribution License 4.0 International License

\begin{abstract}
The aim of this paper is to apply the necessary and sufficient conditions of well-known Lagrangian equations with time dependent case for Minkowski 4-space. Many fundamental geometrical properties for time dependent Minkowski 4-space have been obtained in this paper. The energy equations have been applied to the numerical example in order to test its performance. In the numerical examples, we have studied with two time parameters (earth and space time) for accordance to Minkowski 4-space coordinates. This idea is an interesting approach to energy function with Earth-time and Space-time in physical comment. Moreover, velocity and two time dimensions for energy movement equations have been presented a new concept. This study show some physical application of those equations and interpretations are made in Minkowski space too. Results showed that Lagrangian functions for any surface are same type and depend on time coordinates.
\end{abstract}

Keywords Minkowski 4-space, Jet Bundle, Lagrangian Mechanical System, Euler-Lagrange Equation

\section{Introduction}

Many of the problems in classical mechanics may be solved based on Lagrangian energy equations using Euclidian space, but none of them are calculated on the Minkowski 4-space. Metric structure of Minkowski-4 space different from Euclidian space. For that reason, to solve mechanical problems on jet bundles is difficult. Therefore, this study obtains coordinates Minkowski 4-space by forming jet bundles at first. Second energy equations are solved using a mentioned equations.

In mathematical physics, Minkowski spacetime is a combination of the three-dimensional Euclidean space and time into a four dimensional manifold. Higher dimensional spaces have since became one of the foundations for formally expressing modern mathematics and physics. Time is divided into world time and spacetime. As the union of these times with Euclidean space, it is convenient to use Minkowski 4-space in mathematical physics. The generalization of classical spacetime is permanently a subject of the contemporary mathematical investigations as we can see for example in the references $[1,7,8]$. To follow these research in physics as a first step, we prefer to solve Lagrangian energy equations on the Minkowski 4-space which is based on a jet bundle structure.Works on generalized Minkowski spaces in the references [2, 3, 12], the time-space manifolds and studies for time-dependent Lagrangians can be seen in the references [4, 17].

Inclusion of time dimension for solving Lagrangian energy equations on Minkowski 4-space is an important parameter that improves the Lagrangian system for which we propose to take time derivative coordinates on Jet bundle.

The constraint, real, complex and Para-complex structures on the time-dependent Lagrangian systems can be researched in [7], [13] and [16].

Aycan [7] proved that the jet bundle structures are not changed for Lagrangian energy equations. But, since jet bundle structure included time dimension, it may be easy to solve energy equations with this parameter.Furthermore Aycan and Dagli [8] improved Lagrangian energy equations on complex jet bundles. Also, the presented method in [8] has easily indicate Lagrangian mechanism formulation on a space which has a complex dimension. 
Lagrangian equations are solved with real bundles by [14] and [16]. Minkowski 3-space and its geometrical properties were researched in [5]. Mechanical systems with time parameter were investigated in [9] and [10]. But none of them as far as our knowledge not solved with Minkowski jet bundles using time dimension and time derivatives on coordinates in Minkowski 4-space.

The brief introduction of Lagrangian systems are given in the following way.

If $\mathrm{Q}$ is an m-dimensional configuration manifold and $L: T Q \rightarrow R$ is a regular Lagrangian function, then there is a unique vector field $X$ on $T Q$ and $w$ is a 2 -form on $T^{*} Q$, such that

$$
i_{X_{L}} w_{L}=d E_{L}
$$

where $E_{L}$ is energy associated to $L$ ([14] and [16]). The so-called Euler-Lagrange vector field $X$ is a semi-spray or second order differential equation on $Q$ since its integral curves are the solutions of the Euler-Lagrange equations ([14] and [16]). The triple $\left(T Q, w_{L}, L\right)$ is called Lagrangian system on the tangent bundle $T Q$ [15].

Let, $L: R \times T Q=J(R, Q) \rightarrow R$ and $T Q=\{t\} \times T Q$ be Lagrangian function. The coordinate system on $T Q$ is $\left\{q_{i}, v_{i}\right\}$.

The Poincare cartan 1-form on the $T^{*} Q$ associated with $L$ is;

$$
\begin{aligned}
\alpha_{L} & =d_{J} L+L d v_{i} \\
& =\frac{\partial L}{\partial v_{i}} d q_{i}+L d v_{i}
\end{aligned}
$$

The Poincare cartan 2-form associated with $L$ is

$$
\Omega_{L}=d d_{J} L+d L \wedge d v_{i}
$$

If the paths of semisprays verify the following expressions;

$$
\frac{d}{d t}\left(\frac{\partial L}{\partial v_{i}}\right)-\frac{\partial L}{\partial q_{i}}=0
$$

1.2 is called as Euler-Lagrange equation.

\section{Bundles on Minkowski 4-Space}

Let $(E, \pi, M)$ is a bundle where $E$ and $M$ are manifolds and $\pi: E \rightarrow M$ is a surjective submersion. $E$ is called the total space, $\pi$ is the the projection and $M$ is the base space. This bundle denoted by $\pi$ or $E$. The first jet manifold of $\pi$ is the set $\left\{J_{p}^{1} \phi: p \in M, \phi \in \Gamma_{p}(\pi)\right\}$ and denoted by $J^{1} E$. Here, $\phi: M \rightarrow E \phi$ is a map and called as section of $\pi$. If it is satisfies the condition $\pi \circ \phi=i d_{M}$, then the set of all sections of $\pi$ will be denoted $\Gamma(\pi)$.

Let $(U, u)$ be an adapted coordinate system on $E$, where $u=\left(x_{i}, u_{\alpha}\right)$. The induced coordinate system $\left(U^{1}, u^{1}\right)$ on $J^{1} E$ is defined by

$$
\begin{aligned}
U^{1} & =\left\{J_{p}^{1} \phi: \phi(p) \in U\right\} \\
u^{1} & =\left(x_{i}, u_{\alpha}, u_{\alpha}^{i}\right)
\end{aligned}
$$

where $x_{i}\left(J_{p}^{1} \phi\right)=x_{i}(p), u_{\alpha}\left(J_{p}^{1} \phi\right)=u_{\alpha}(\phi(p))$ and new functions $u_{\alpha}^{i}: U^{1} \rightarrow R$ are specified by $u_{\alpha}^{i}\left(J_{p}^{1} \phi\right)=\frac{\partial \phi_{\alpha}}{\partial x_{i}}(p)$ and are known as derivative coordinates [7]. Using those coordinate system, the following coordinate system are proposed to Minkowski 4-space.

Let the bundle structure $\left(E_{1}^{4}, \pi, R\right)$ and the coordinates of the manifold $E_{1}^{4}$ are $\left(x_{1}, x_{2}, x_{3}, x_{4}\right)$, the coordinate of the manifold $R$ is $(t)$. In addition the coordinates of the manifold $J^{1} E_{1}^{4}$ are $\left(t, x_{1}, x_{2}, x_{3}, x_{4} \dot{x_{1}}, \dot{x_{2}}, \dot{x_{3}}, \dot{x_{4}}\right)$.

Then derivative coordinates are writen as

$$
\dot{x}_{i}=\frac{d x_{i}}{d t} \text {. }
$$

\section{Lagrangian Mechanical Systems For Minkowski Space with Jet Bundle}

The Minkowski 4-space $E_{1}^{4}$ is the Euclidean 4-space $E^{4}$ provided that the standard metric given by

$$
g=-d x_{1}^{2}+d x_{2}^{2}+d x_{3}^{2}+d x_{4}^{2}
$$


where $\left(x_{1}, x_{2}, x_{3}, x_{4}\right)$ is a rectangular coordinate system of $E^{4}$. Here, $g$ denoted the metric construction and $d$ is the differential form. Since $g$ is an indefinite metric, vector $v \in E_{1}^{4}$ is one of three Lorentzian characters; it can be space -like if $g(v, v)>0$ or $g(v)=0$, time-like if $g(v, v)<0$ and null if $g(v)=0$ and $v \neq 0$. Similarly, an arbitrary curve $\alpha=\alpha(s)$ in $E_{1}^{4}$ can locally be space-like, time-like, or null(light-like), if all of its velocity vectors $\alpha$ ' are respectively space-like, time-like or null, for every $s$.

$\tau$ is the set of all time-like vectors in $E_{1}^{4}$. For $\forall u \in \tau$; the set

$$
C(\vec{u})=\{\vec{x} \in \tau:\langle\vec{u}, \vec{x}\rangle<0\}=\left\{\vec{x} \in E_{1}^{4}: g(x-u, x-u)<0\right\}
$$

defined as timecone [1] and [11].

Theorem 1. The time-like vectors $\vec{x}$ and $\vec{y}$ in Minkowski 4-space $E_{1}^{4}$ are in the same timecone (Figure-1),

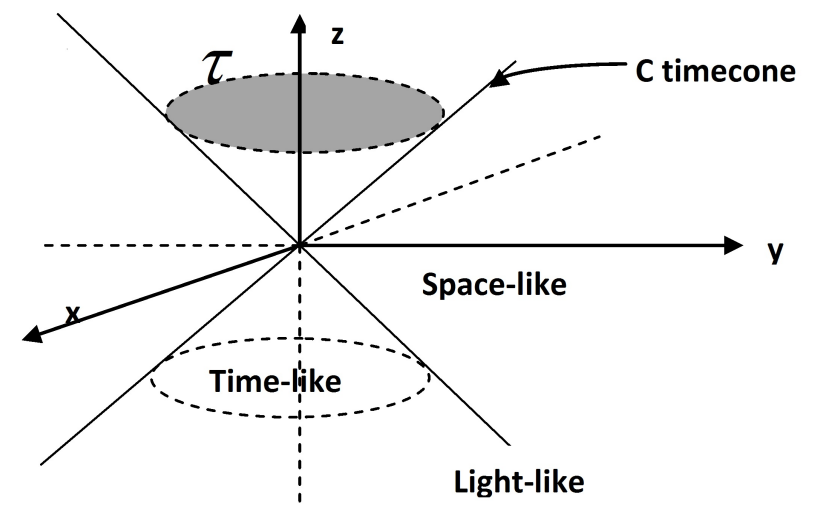

Figure 1. Figure-1,timecone

$\langle\vec{x}, \vec{y}\rangle=-\|\vec{x}\|\|\vec{y}\| \cosh \theta$ and here $\theta$ is the Lorentz time-like angle between $\vec{x}$ and $\vec{y}$ vectors.

Definition 1. Let $J$ is a tensor field of type first-order covariant and first-order contra-variant such that $J: T\left(J^{1} E_{1}^{4}\right) \longrightarrow$ $T\left(J^{1} E_{1}^{4}\right)$ by

$$
J\left(\frac{\partial}{\partial x_{1}}\right)=-\frac{\partial}{\partial \dot{x}_{1}}, J\left(\frac{\partial}{\partial x_{2}}\right)=\frac{\partial}{\partial \dot{x}_{2}}, J\left(\frac{\partial}{\partial x_{3}}\right)=\frac{\partial}{\partial \dot{x}_{3}}, J\left(\frac{\partial}{\partial x_{4}}\right)=\frac{\partial}{\partial \dot{x}_{4}}
$$

$J\left(\frac{\partial}{\partial \dot{x_{1}}}\right)=J\left(\frac{\partial}{\partial \dot{x_{2}}}\right)=J\left(\frac{\partial}{\partial \dot{x_{3}}}\right)=J\left(\frac{\partial}{\partial \dot{x_{4}}}\right)=0$

$J\left(\frac{\partial}{\partial t}\right)=-\dot{x}_{1} \frac{\partial}{\partial \dot{x_{1}}}+\dot{x_{2}} \frac{\partial}{\partial \dot{x_{2}}}+\dot{x_{3}} \frac{\partial}{\partial \dot{x_{3}}}+\dot{x_{4}} \frac{\partial}{\partial \dot{x_{4}}}$

$J$ can be calculated as a tensor field from 3.1, as

$$
\begin{aligned}
J= & \left(-d x_{1}-\dot{x_{1}} d t\right) \times \frac{\partial}{\partial \dot{x_{1}}}+\left(d x_{2}+\dot{x_{2}} d t\right) \times \frac{\partial}{\partial \dot{x_{2}}} \\
& +\left(d x_{3}+\dot{x_{3}} d t\right) \times \frac{\partial}{\partial \dot{x_{3}}}+\left(d x_{4}+\dot{x_{4}} d t\right) \times \frac{\partial}{\partial \dot{x_{4}}}
\end{aligned}
$$

This tensor field is the almost tangent structure and especially $J^{2}=0$.

A semi-spray is a vector field over $E_{1}^{4}$ and defined as below;

$$
\begin{aligned}
\varepsilon= & \frac{\partial}{\partial t}-\dot{x_{1}} \frac{\partial}{\partial x_{1}}+\dot{x_{2}} \frac{\partial}{\partial x_{2}}+\dot{x_{3}} \frac{\partial}{\partial x_{3}}+\dot{x_{4}} \frac{\partial}{\partial x_{4}} \\
& -\varepsilon_{1} \frac{\partial}{\partial \dot{x_{1}}}+\varepsilon_{2} \frac{\partial}{\partial \dot{x_{2}}}+\varepsilon_{3} \frac{\partial}{\partial \dot{x_{3}}}+\varepsilon_{4} \frac{\partial}{\partial \dot{x_{4}}}
\end{aligned}
$$

By calculate $J(\varepsilon)$, then equation 3.4 are found

$$
V=J \varepsilon=-2 \dot{x_{1}} \frac{\partial}{\partial \dot{x_{1}}}+2 \dot{x_{2}} \frac{\partial}{\partial \dot{x_{2}}}+2 \dot{x_{3}} \frac{\partial}{\partial \dot{x_{3}}}+2 \dot{x_{4}} \frac{\partial}{\partial \dot{x_{4}}}
$$


which is called "Liouville vector field".

Moreover, "Poincare-Cartan 1-form" is written as:

$$
\begin{gathered}
\alpha_{L}=d_{J} L+L d t \\
\alpha_{L}=-\dot{x_{1}} \frac{\partial L}{\partial \dot{x_{1}}} d t+\dot{x_{2}} \frac{\partial L}{\partial \dot{x_{2}}} d t+\dot{x_{3}} \frac{\partial L}{\partial \dot{x_{3}}} d t+\dot{x_{4}} \frac{\partial L}{\partial \dot{x_{4}}} d t \\
-\frac{\partial L}{\partial \dot{x_{1}}} d x_{1}+\frac{\partial L}{\partial \dot{x_{2}}} d x_{2}+\frac{\partial L}{\partial \dot{x_{3}}} d x_{3}+\frac{\partial L}{\partial \dot{x_{4}}} d x_{4}+L d t
\end{gathered}
$$

Then we can write differential operator $d$,

$$
\begin{aligned}
d= & \frac{\partial}{\partial t} d t-\frac{\partial}{\partial x_{1}} d x_{1}+\frac{\partial}{\partial x_{2}} d x_{2}+\frac{\partial}{\partial x_{3}} d x_{3}+\frac{\partial}{\partial x_{4}} d x_{4} \\
& -\frac{\partial}{\partial \dot{x_{1}}} d \dot{x_{1}}+\frac{\partial}{\partial \dot{x_{2}}} d \dot{x_{2}}+\frac{\partial}{\partial \dot{x_{3}}} d \dot{x_{3}}+\frac{\partial}{\partial \dot{x_{4}}} d \dot{x_{4}}
\end{aligned}
$$

By differentiating $\alpha_{L}$ to d, "Poincare-Cartan 2-form" is obtained by

$$
\Omega_{L}=d d_{J} L+d L \wedge d t
$$

But, for this writting, we can assume notations for negative terms. We denote this notations as follows;

$$
\begin{aligned}
& \delta_{i}=\delta_{j}=\left\{\begin{array}{cc}
-1, \quad i=1 \\
1, \quad i=2,3,4
\end{array}\right. \\
& \delta_{i j}=\left\{\begin{array}{cc}
-1, \quad i=j=1 \\
1, & i \geq 2 \text { ve } i=j \\
-1, & i \geq 2 \text { ve } i \neq j
\end{array}\right. \\
& \mu_{i j}=\left\{\begin{array}{cc}
0, & i=j \\
-1, & i \neq j
\end{array}\right.
\end{aligned}
$$

so

$$
\begin{aligned}
\Omega_{L} & =\sum_{i=1}^{4} \delta_{i}\left\{\left[\left(d x_{i} \wedge d t\right)\left(\frac{\partial^{2} L}{\partial t \partial \dot{x_{i}}}+\sum_{j=1}^{4} \delta_{j} \dot{x_{j}} \frac{\partial^{2} L}{\partial x_{i} \partial \dot{x_{j}}}\right)\right]\right. \\
& \left.+\left[\left(d \dot{x_{i}} \wedge d t\right)\left(\sum_{j=1}^{4} \delta_{j} \dot{x_{j}} \frac{\partial^{2} L}{\partial \dot{x_{i} \partial \dot{x_{j}}}}\right)\right]+\left(\frac{\partial L}{\partial x_{i}}+2 \frac{\partial L}{\partial \dot{x_{i}}}\right)\right\} \\
& +\sum_{i, j=1}^{4}\left[\left(d \dot{x_{i}} \wedge d \dot{x_{j}}\right) \delta_{i j} \frac{\partial^{2} L}{\partial \dot{x_{i}} \partial x_{j}}+\left(d x_{i} \wedge d x_{j}\right) \mu_{i j} \frac{\partial^{2} L}{\partial x_{i} \partial \dot{x_{j}}}\right]+2 \frac{\partial L}{\partial \dot{x_{1}}}
\end{aligned}
$$

Definition 2. Solutions of the Euler-Lagrange equation can be found by assuming $i_{\varepsilon} \Omega_{L}=\Omega_{L}(\varepsilon)=0$.

$$
\begin{aligned}
i_{\varepsilon} \Omega_{L}= & \Omega_{L}(\varepsilon) \\
= & -\sum_{i=1}^{4} \dot{x_{i}} \frac{\partial^{2} L}{\partial t \partial \dot{x_{i}}}+\sum_{i=1}^{4} \delta_{i} \dot{x_{i}}\left(\sum_{j=1}^{4} \dot{x_{j}} \frac{\partial^{2} L}{\partial \dot{x_{i} \partial x_{j}}}+\sum_{j=1}^{4} \varepsilon_{j} \frac{\partial^{2} L}{\partial \dot{x_{i} \partial \dot{x_{j}}}}\right) \\
& +\sum_{i=1}^{4}\left(\dot{x_{i}} \frac{\partial L}{\partial \dot{x_{i}}}+2 \delta_{i} \varepsilon_{i} \frac{\partial L}{\partial \dot{x_{i}}}\right)+2 \varepsilon_{1} \frac{\partial L}{\partial \dot{x_{1}}} d t
\end{aligned}
$$




$$
\begin{aligned}
& +\left\{\sum_{i=1}^{4} \delta_{i}\left(\frac{\partial^{2} L}{\partial t \partial \dot{x_{i}}}-\frac{\partial L}{\partial \dot{x_{i}}}\right)+\sum_{i, j=1}^{4}\left(\delta_{i} \varepsilon_{j} \frac{\partial^{2} L}{\partial \dot{x_{i} \partial \dot{x_{j}}}}+\mu_{i j} \dot{x_{i}} \frac{\partial^{2} L}{\partial x_{i} \partial \dot{x_{j}}}\right)\right. \\
& +\sum_{i, j=1}^{4} \delta_{i j} \delta_{i} \dot{x_{j}} \frac{\partial^{2} L}{\partial \dot{x_{j} \partial x_{i}}} d x_{i}-\left\{\sum_{i, j=1}^{4} \delta_{i j}\left(\dot{x_{j}} \frac{\partial^{2} L}{\partial \dot{x_{i} \partial \dot{x_{j}}}}+\dot{x_{j}} \frac{\partial^{2} L}{\partial \dot{x_{i} \partial \dot{x_{j}}}}\right)\right\} d \dot{x_{i}}
\end{aligned}
$$

By equalizing equation 3.8 to zero, then 3.9 are obtained.

$$
\begin{aligned}
& I:-\frac{\partial^{2} L}{\partial t \partial \dot{x_{1}}}+\frac{\partial L}{\partial x_{1}}\left(\sum_{i=1}^{4} \delta_{i} \dot{x_{i}} \frac{\partial L}{\partial \dot{x_{i}}}+L\right)-\frac{\partial}{\partial \dot{x_{1}}}\left(\sum_{i=1}^{4} \varepsilon_{i} \frac{\partial L}{\partial \dot{x_{i}}}\right) \\
& +\sum_{i=1, j=1}^{4} \mu_{i j} \dot{x}_{j}\left(\frac{\partial^{2} L}{\partial x_{i} \partial \dot{x_{j}}}-\frac{\partial^{2} L}{\partial x_{j} \partial \dot{x_{i}}}\right)=0 \\
& \text { II: } \frac{\partial^{2} L}{\partial t \partial \dot{x_{2}}}-\frac{\partial L}{\partial x_{2}}\left(\sum_{i=1}^{4} \delta_{i} \dot{x_{i}} \frac{\partial L}{\partial \dot{x_{i}}}+L\right)+\frac{\partial}{\partial \dot{x_{2}}}\left(\sum_{i=1}^{4} \varepsilon_{i} \frac{\partial L}{\partial \dot{x_{i}}}\right) \\
& -\sum_{i=2, j=1}^{4} \mu_{i j} \dot{x}_{j}\left(\frac{\partial^{2} L}{\partial x_{i} \partial \dot{x_{j}}}-\frac{\partial^{2} L}{\partial x_{j} \partial \dot{x_{i}}}\right)=0 \\
& \text { III: } \frac{\partial^{2} L}{\partial t \partial \dot{x_{3}}}-\frac{\partial L}{\partial x_{3}}\left(\sum_{i=1}^{4} \delta_{i} \dot{x_{i}} \frac{\partial L}{\partial \dot{x_{i}}}+L\right)+\frac{\partial}{\partial \dot{x_{3}}}\left(\sum_{i=1}^{4} \varepsilon_{i} \frac{\partial L}{\partial \dot{x_{i}}}\right) \\
& -\sum_{i=3, j=1}^{4} \mu_{i j} \dot{x}_{j}\left(\frac{\partial^{2} L}{\partial x_{i} \partial \dot{x_{j}}}-\frac{\partial^{2} L}{\partial x_{j} \partial \dot{x_{i}}}\right)=0 \\
& I V: \frac{\partial^{2} L}{\partial t \partial \dot{x_{4}}}-\frac{\partial L}{\partial x_{4}}\left(\sum_{i=1}^{4} \delta_{i} \dot{x_{i}} \frac{\partial L}{\partial \dot{x_{i}}}+L\right)+\frac{\partial}{\partial \dot{x_{4}}}\left(\sum_{i=1}^{4} \varepsilon_{i} \frac{\partial L}{\partial \dot{x_{i}}}\right) \\
& -\sum_{i=4, j=1}^{4} \mu_{i j} \dot{x}_{j}\left(\frac{\partial^{2} L}{\partial x_{i} \partial \dot{x}_{j}}-\frac{\partial^{2} L}{\partial x_{j} \partial \dot{x_{i}}}\right)=0
\end{aligned}
$$

$V I:-\frac{\partial}{\partial \dot{x_{2}}}\left(\sum_{i=1}^{4} \delta_{i} \dot{x_{i}} \frac{\partial L}{\partial \dot{x_{i}}}+L\right)-\frac{\partial}{\partial \dot{x_{2}}}\left(\sum_{i=1}^{4} \dot{x_{i}} \frac{\partial L}{\partial \dot{x_{i}}}+L\right)=0$

$$
\text { VII: }-\frac{\partial}{\partial \dot{x_{3}}}\left(\sum_{i=1}^{4} \delta_{i} \dot{x_{i}} \frac{\partial L}{\partial \dot{x_{i}}}+L\right)+\frac{\partial}{\partial \dot{x_{3}}}\left(\sum_{i=1}^{4} \dot{x_{i}} \frac{\partial L}{\partial \dot{x_{i}}}+L\right)=0
$$

VIII: $-\frac{\partial}{\partial \dot{x_{4}}}\left(\sum_{i=1}^{4} \delta_{i} \dot{x_{i}} \frac{\partial L}{\partial \dot{x_{i}}}+L\right)+\frac{\partial}{\partial \dot{x_{4}}}\left(\sum_{i=1}^{4} \dot{x_{i}} \frac{\partial L}{\partial \dot{x_{i}}}+L\right)=0$

$$
I X:\left\{-\sum_{i=1}^{4} \dot{x_{i}} \frac{\partial^{2} L}{\partial t \partial \dot{x}_{i}}+\sum_{i=1}^{4} \delta_{i} \dot{x_{i}}\left(\sum_{j=1}^{4} \dot{x_{j}} \frac{\partial^{2} L}{\partial \dot{x}_{i} \partial x_{j}}+\sum_{j=1}^{4} \varepsilon_{j} \frac{\partial^{2} L}{\partial \dot{x}_{i} \partial \dot{x_{j}}}\right)\right.
$$




$$
\left.+\sum_{i=1}^{4}\left(\dot{x_{i}} \frac{\partial L}{\partial \dot{x_{i}}}+2 \delta_{i} \varepsilon_{i} \frac{\partial L}{\partial \dot{x_{i}}}\right)+2 \varepsilon_{1} \frac{\partial L}{\partial \dot{x_{1}}}\right\}=0
$$

3.9 represents a non-linear equations system. For solution of this non-linear equations system, we can assume a special initial condition as follows;

$$
\varepsilon_{1}=-\dot{x_{1}}, \quad \varepsilon_{2}=\dot{x}_{2}, \quad \varepsilon_{3}=\dot{x_{3}}, \quad \varepsilon_{4}=\dot{x} x_{4}
$$

In equation 3.10, first term must be negative, because, Minkowski metric is defined as $(-,+,+,+)$ in this study. Then following equalities can be hold;

$$
\dot{x_{1}}(I)-\dot{x_{2}}(I I)-\dot{x_{3}}(I I I)-\dot{x_{4}}(I V)+\dot{x_{1}}(V)-\dot{x_{2}}(V I)-\dot{x_{3}}(V I I)-\dot{x_{4}}(V I I I)+(I X)=0
$$

Solving 3.11 the following equation can be obtained. We can write 3.11 in a general form as follows,

$$
\begin{aligned}
& -\frac{\partial}{\partial t}\left(\sum_{i=1}^{4} \dot{x_{i}} \frac{\partial L}{\partial \dot{x_{i}}}\right)-\dot{x_{1}} \frac{\partial L}{\partial \dot{x}_{1}}\left(\delta_{i} \sum_{i=1}^{4} \dot{x_{i}} \frac{\partial L}{\partial \dot{x}_{i}}+\sum_{i=1}^{4} \dot{x_{i}} \frac{\partial L}{\partial \dot{x}_{i}}\right) \\
& +\left(\delta_{i} \sum_{i=1}^{4} \dot{x_{i}} \frac{\partial L}{\partial \dot{x_{i}}}\right)\left(\sum_{j=1}^{4} \dot{x_{j}} \frac{\partial L}{\partial x_{j}}\right)+\left(\sum_{i=1}^{4} \dot{x_{i}} \frac{\partial L}{\partial \dot{x_{i}}}\right)\left(\sum_{j=1}^{4} \dot{x_{j}} \frac{\partial L}{\partial \dot{x_{j}}}\right) \\
& +\left(\delta_{i} \sum_{i=1}^{4} \dot{x_{i}} \frac{\partial L}{\partial \dot{x_{i}}}+\sum_{i=1}^{4} \dot{x_{i}} \frac{\partial L}{\partial \dot{x_{i}}}\right)+\sum_{i=1}^{4} \dot{x_{i}} \frac{\partial L}{\partial x_{i}}=0
\end{aligned}
$$

This is Euler-Lagrange equation and its solution is the semi-spray on the bundle $J^{1} E_{1}^{4}$.

Following examples show an application of equation 3.12 .

Example 1. We are analyzing the energy emerging by the movement of a particle ' $m$ '. First, we assume that this particle in the space. It moves on a space-like curve towards to earth. If it falls on the earth surface, it will be on a null vector in Minkowski timecone. Then its movement continues on a time-like vector. Also, we can accept the movement in timecone occurs on a helix curve, which is lay in timecone. For examine the occurence energy for this movement, we can constitute jet bundle structure for this helix for time parameter. On this cone, when it can be said for the time past or future, then it can be studied only time-like vectors.Also, first jets must be time-like vectors. Because, space-like and null vectors define in this space, but it can't define continuity the movement of the particle. The event must be materializing in timecone, also the vectors, which we use, can be time-like vectors. Now, we take into account the helix curve in the cone.

Figure-2 denoted the helix curve on the sphere in Minkowski 4-space,

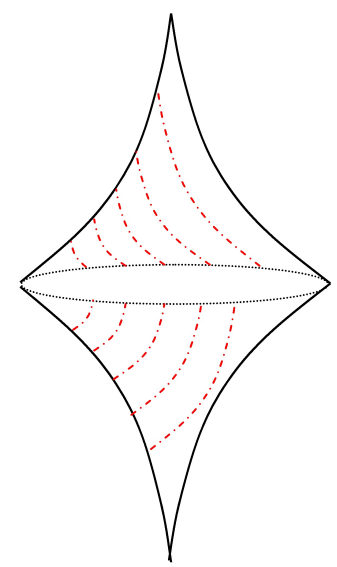

Figure 2. Figure-2,helix on timecone.

Figure-3 denoted the movement our particle. 


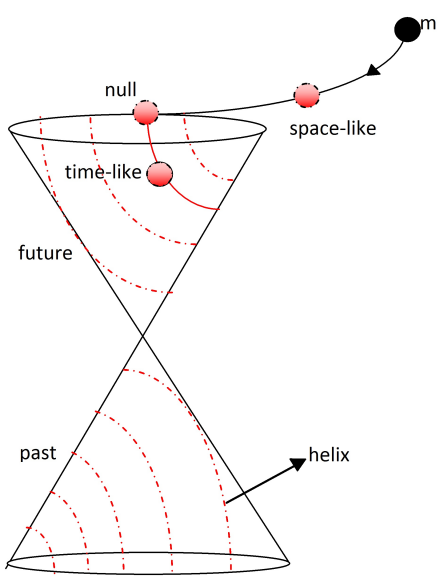

Figure 3. Figure-3, Movement of article on Minkowski helix

Minkowski helix is defined as $(r \sinh u \theta, r \cosh u \theta \sin \theta, r \cosh u \theta \cos \theta)$ [1]. Here $r$ is radius function and related to time parameter, $r=r(t) . \theta$ is the angle, which is between tangent vector and curve in all points and it is a fixed angle. If this helix curve be time-like, then it must be provided the inequality $\left\langle\alpha^{\prime}, \alpha^{\prime}\right\rangle\langle 0$ and the angle $\theta$ in two time-like vectors can be defined with equality $\langle\vec{x}, \vec{y}\rangle=-\|\vec{x}\|\|\vec{y}\| \cosh \theta$. The velocity vector for this curve is

$\alpha^{\prime}(\theta)=(r \sinh u \theta, r \cosh u \theta \sin \theta, r \cosh u \theta \cos \theta)^{\prime}$

$=u r \cosh u \theta, u r \sinh u \theta \sin \theta+r \cosh u \theta \cos \theta, u r \sinh u \theta \cos \theta-r \cosh u \theta \sin \theta)$

If this curve is time-like, then it will satisfy following equality

$$
\left\langle\alpha^{\prime}, \alpha^{\prime}\right\rangle=r^{2}\left(\cosh ^{2} u \theta-u^{2}\right)
$$

So, parameter $u$ can be provided the inequality

$$
-u<\cosh u \theta<u
$$

The jet bundle coordinates for this helix are

$(s, t, r \sinh u \theta, r \cosh u \theta \sin \theta, r \cosh u \theta \cos \theta$,

$\dot{t}, \dot{r} \sinh u \theta, \dot{r} \cosh u \theta \sin \theta, \dot{r} \cosh u \theta \cos \theta)$

Here $s$ is a space-time parameter, $t$ is earth-time parameter. In this example, for the harmony with the number jet bundle coordinates in Minkowski 4-space $E_{1}^{4}$ and for the physical comment, we must accept two-time parameter. On the other hand, this is a reality, because time for space and earth different each other. With using this coordinates and with simplication in terms in the equation 3.12, we can obtain Euler-Lagrange equation for this helix as follows;

$$
\begin{aligned}
& -\dot{t} \frac{\partial^{2} L}{\partial s \partial \dot{t}}-3 \dot{r} \frac{\partial^{2} L}{\partial s \partial \dot{r}}-\dot{t}^{2} \frac{\partial^{2} L}{\partial t \partial \dot{t}}+3 \dot{t} \dot{r} \frac{\partial^{2} L}{\partial t \partial \dot{r}}+\dot{t} \frac{\partial L}{\partial t} \\
& -3 \dot{t} \dot{r} \frac{\partial^{2} L}{\partial r \partial \dot{t}}+9 \dot{r}^{2} \frac{\partial^{2} L}{\partial r \partial \dot{r}}+3 \dot{r} \frac{\partial L}{\partial r}+\dot{t}^{2} \frac{\partial^{2} L}{\partial t \partial \dot{t}} \\
& +3 \dot{t} \dot{r} \frac{\partial^{2} L}{\partial \dot{r} \partial \dot{t}}+9 \dot{r}^{2} \frac{\partial^{2} L}{\partial \dot{r} \partial \dot{r}}-3 \dot{t} \dot{r} \frac{\partial^{2} L}{\partial \dot{r} \partial \dot{t}}+6 \dot{r} \frac{\partial L}{\partial \dot{r}}=0
\end{aligned}
$$

Now, we calculate the solution of this Euler-Lagrange equation. Here, the Lagrange function is $L=L(r, t)$ and radius function is $r=r(t)$; so Lagrange function is connected with time and radius parameters, radius is connected with time parameter.

For calculating this equation, we consider

$$
\frac{d t}{d s}=k, \quad(k \text { is a constant })
$$


It can be a fixed relation in space-time and earth-time parameters. Furthermore, this assumption is not enough alone. Hence, we consider

$\frac{d L}{d \dot{r}}=\lambda \Rightarrow L=\lambda \dot{r}$ and $\frac{d L}{d \dot{t}}=\mu \Rightarrow L=\mu \dot{t}$.

We know that $L$ energy function include time and radius parameter, and then it included derivative time and radius parameters coming from jet bundle structure too.

With calculation the equation 3.16, we get solution of Lagrange energy function;

$$
L=-\frac{3}{2} \lambda r
$$

Furthermore, radius function

$$
\begin{aligned}
\frac{d r}{d s}=-\frac{3}{2} r & \Rightarrow r=e^{-\frac{3}{2} s} \\
& \Rightarrow r=e^{-\frac{3}{2} \frac{t}{k}}
\end{aligned}
$$

Then the energy Lagrange energy function can writen as

$$
L=-\frac{3}{2} \lambda e^{-\frac{3}{2} \frac{t}{k}}
$$

From 3.18, with our acceptance, time parameter is one of the main parameter. Time has an important role on the occurence energy for the movement. On the other hand, when the ratio of the time in Minkowski and earth space is $k$, the ratio of the speeds in Minkowski and earth space is $k$ too. So we can write,

$$
\frac{\left\|\frac{d \alpha}{d t}\right\|}{\left\|\frac{d \alpha}{d s}\right\|}=\frac{\sqrt{1+\left(\frac{d r}{d t}\right)^{2} \cosh 2 u \theta}}{\sqrt{\left(\frac{d r}{d s}\right)^{2} \cosh 2 u \theta-k^{2}}}=k
$$

and

$$
\frac{\sqrt{1+\frac{9}{4 k^{2}} e^{-\frac{3 t}{k}} \cosh 2 u \theta}}{\sqrt{\frac{9}{4} e^{-3 s} \cosh 2 u \theta-k^{2}}}=k
$$

also we obtain

$$
t=-\frac{k}{3} \ln \left[\frac{4 k^{2}\left(1+k^{4}\right)}{9\left(k^{4}-1\right) \cosh 2 u \theta}\right]
$$

with this conclusion we can calculate the Lagrangian energy in following way. When we write this values in equation 3.19, we calculate $k$ parameters for $\lambda=-1$. Then,

$$
\begin{aligned}
& t=1 \Rightarrow k=-4,302932897 \Rightarrow L=2,125622176 \\
& t=2 \Rightarrow k=-5,336423366 \Rightarrow L=2,631724656 \\
& t=3 \Rightarrow k=-6,251374542 \Rightarrow L=2,149823941 \\
& t=4 \Rightarrow k=-7,093059294 \Rightarrow L=3,495101613
\end{aligned}
$$

This is showed that, when time is larger, the Lagrangian energy values are almost same. Lagrangian energy values are accelerate slowly, because the movement on the helix curve are occured regularly. 


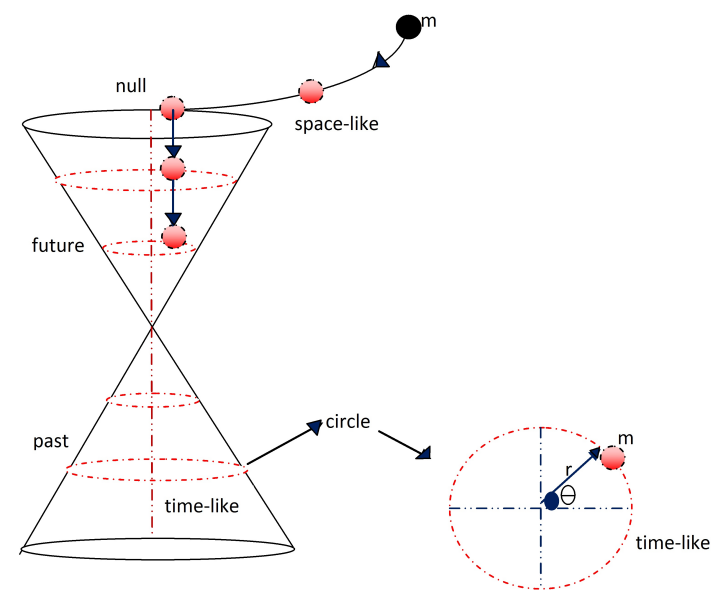

Figure 4. Figure 4. Circle on timecone

Example 2. In this example, we define a circle in timecone (Figure-4).And, we are analyzing the movement of the particle ' $m$ ' in this circle. For this reason we want to define the jet bundle structure for the circle. The jet bundle coordinates are,

$$
(t, r \cos \theta, r \sin \theta, u, \dot{r} \cos \theta, \dot{r} \sin \theta, 0)
$$

Here $s$ is a space-time parameter, $t$ is earth-time parameter. On the other hand it can be seen from the figure-4, this circle exist in any plane on the $z$ axis. From this the paramater $u$, has a constant value. With using this coordinates and with simplication in terms in the equation 3.12, we can obtain Euler-Lagrange equation for this circle as follows;

$$
\begin{aligned}
& -\dot{t} \frac{\partial^{2} L}{\partial s \partial \dot{t}}-2 \dot{r} \frac{\partial^{2} L}{\partial s \partial \dot{r}}-\dot{t}^{2} \frac{\partial^{2} L}{\partial t \partial \dot{t}}+2 \dot{t} \dot{r} \frac{\partial^{2} L}{\partial t \partial \dot{r}}+\dot{t} \frac{\partial L}{\partial t} \\
& -2 \dot{t} \dot{r} \frac{\partial^{2} L}{\partial r \partial \dot{t}}+4 \dot{r}^{2} \frac{\partial^{2} L}{\partial r \partial \dot{r}}+2 \dot{r} \frac{\partial L}{\partial r}+\dot{t}^{2} \frac{\partial^{2} L}{\partial \dot{t} \partial \dot{t}} \\
& +2 \dot{t} \dot{r} \frac{\partial^{2} L}{\partial \dot{r} \partial \dot{t}}+4 \dot{r}^{2} \frac{\partial^{2} L}{\partial \dot{r} \partial \dot{r}}-2 \dot{t} \dot{r} \frac{\partial^{2} L}{\partial \dot{r} \partial \dot{t}}+4 \dot{r} \frac{\partial L}{\partial \dot{r}}=0
\end{aligned}
$$

Here we assume that

$$
\frac{d t}{d s}=k, \quad(k \text { is a constant })
$$

and

$$
\begin{aligned}
& \frac{d L}{d \dot{r}}=\lambda \Rightarrow L=\lambda \dot{r} \\
& \frac{d L}{d \dot{t}}=\mu \Rightarrow L=\mu \dot{t}
\end{aligned}
$$

too. Also, the following equation is obtained in similarly way

$$
L=-\frac{4}{3} \lambda r
$$

Futhermore

$$
\begin{aligned}
\frac{d r}{d s}=-\frac{4}{3} r & \Rightarrow r=e^{-\frac{4}{3} s} \\
& \Rightarrow r=e^{-\frac{4}{3} \frac{t}{k}}
\end{aligned}
$$

Then,

$$
L=-\frac{4}{3} \lambda e^{-\frac{4}{3} \frac{t}{k}}
$$

If it is noticed that, Lagrange function in 3.18 and 3.23 is similar. The difference between this equations is the constant term in exponential phase. Also, we can calculate the Lagrange energy in the similar way with example one. Here we obtain 


$$
\frac{\left\|\frac{d \alpha}{d t}\right\|}{\left\|\frac{d \alpha}{d s}\right\|}=\frac{\sqrt{1+\left(\frac{d r}{d t}\right)^{2}}}{\sqrt{\left(\frac{d r}{d s}\right)^{2}-k^{2}}}=k
$$

and

$$
\frac{\sqrt{1+\frac{16}{9 k^{2}} e^{-\frac{8 t}{3 k}}}}{\sqrt{\frac{16}{9} e^{-\frac{8}{3} s}-k^{2}}}=k
$$

so

$$
t=-\frac{3 k}{8} \ln \left[\frac{9 k^{2}\left(1+k^{4}\right)}{16\left(k^{4}-1\right)}\right]
$$

Then, with this conclusion we can the Lagrangian energy for circle in following way. When we write time values in equation 3.24, we can calculate $k$ parameters for $\lambda=-1$. Also,

$$
\begin{aligned}
& t=1 \Rightarrow k=-2,297972884 \Rightarrow L=2,38191218 \\
& t=2 \Rightarrow k=-3,109688970 \Rightarrow L=3,14312310 \\
& t=3 \Rightarrow k=-1,00009442, \Rightarrow L=72,77004149 \\
& t=4 \Rightarrow k=-1,000006557 \Rightarrow L=276,1600076
\end{aligned}
$$

It is showed that, when time is larger, then Lagrangian energy values increase very fast. Because, the mobile object on the circle falls free, also this object speed up very quickly. Finally, it arrives the speed of light.

\section{Conclusion and Discussion}

This study investigates the possible enhancement of Lagrangian equations on Minkowski space. Furthermore, in a different space model, jet bundle structure on Minkowski 4-manifold has been constitued in this paper. This bundle has been generated a form from real bundle structure. The application of energy equations with respect to two time parameter have been taken into account on jet bundles on the helix curve and on the circle on timecone provide for testing the solution methods. The following results can be droven from this study.

1)Lagrangian energy equation for Minkowski 4-space can be improved by taking into account time dimension using jet bundles.

2)Explanation about negative defined metric form for Lagrange equations on Minkowski 4-space using jet bundles are corresponded to equation 3.12, that leades to a general form of Lagrange equation in 1.2. The main difference in this equations is the negative term coming from Minkowski metric and derivative coordinates coming from Jet bundle structure. If this metric takes positive in all terms, then we can calculate the Euler-Lagrange function in Euclid space in the same form.

3)Given examples showed that proposed non-linear partial differential equations can be solved with respect to special acceptance, which are compatible to physical and mathematical reality.

4)Physical interpretation of this improved Lagrangian equations in this paper may be leaded to further invastigates. This is a interesting study, because this space model is preferred for physicants. One of the interesting conclusion of this study is, when the time is very big, the Lagrangian energy of a movement particle can be seen in a static case. Two time parameter is a reality for a space model. Thus, studying with Minkowski 4-space is a natural phenomena. And this study is a generally research for Lagrangian energy depended on time.

5)As a result of equation 3.18 and 3.23, if a partial is too fast then the Lagrangian energy is a stably state case. Similarly, if time is too long, then the Lagrangian energy is a stably state case. 
6)All examples showed that, Lagrangian energy values increasing in a certain role and approach a fixed value. Obviously, this energy converge the speed of light. In the example one, the movement on the helix curve is a regaularly movement. This movement occurs the form of slip on helix. Also, energy values accelerate slowly. But, in the example two the movement on the circle is a free movement. This movement occurs in the form of falling, so the energy accelerate very speedly. This conclusion is the most striking results of this study.

\section{REFERENCES}

[1] A,Ali, Determination of Time-Like Helices From Intrınsic Equation in Minkowski 3-space, Physics Letter A, 2009

[2] A. G. Horvath, Semi-indefnite Inner Product and Generalized Minkowski spaces, Journal of Geometry and Physics, 60, 1190-1208, 2010

[3] A. G. Horvath, Generalized Minkowski Space With Changing Shape, Aequationes Mathematicae, 87/3, 337-377, 2014

[4] A. G. Horvath, Relativity Theory İn Time-space Manifold, Universal Journal of Physics and Application, 10/4, 115-127, 2016

[5] A., Turgut, 3-Boyutlu Minkowski Uzayında Spacelike ve Timelike Regle Yüzeyler, PhD Thesis, 1995

[6] B. O’neill, Semi-Riemann Geometry with Applications to Relativity, Academic Press, New York, 1983

[7] C.Aycan, The Lifts of Euler-Lgrange and Hamiltonian Equations on the Extended Jet Bundles, D. Sc. Thesis, Osmangazi Univ. , Eskişehir, 2003

[8] C.Aycan, S. Dagli, Improving Lagrangian Energy Equation On The Kahler jet bundles, IJGMMP, V10 N7, August, 2013

[9] G.Giachetta, L. Mangiarotti and G. Sardanashvily, Geometric Quantization of Mechanical systems with time-dependent parameters, J, Math. Phys. 43, 2882-2894, 2002

[10] G. Sardanashvilly, Classical and quantum mechanics with time-dependent parameters J. Math. Phys. 41, 5245-5255, 2000

[11] H. R. Brown, Physical Relativity. Oxford University press, New York, 2005

[12] J. K. Beem, P. E., Ehrlich, Global Lorenzian Geometry, Marcel Dekker Inc., New York, 1981

[13] M. Crampin, On the differential geometry of Euler-Lagrange equations, and the inverse problem of Lagrangian dynamics, J. Phys. A-Math. And Gen., 14/10, 2567-2575, 1981

[14] M. Crampin, Lagrangian sub manifolds and the Euler-Lagrange equations in the higher-order mechanics, Lett. Math. Phys., 19/1 , 53-58, 1990

[15] M. De Leon and P.R. Rodriguez, Methods of Differential Geometry in Analytical Mechanics, North-Hol. Math. St.,152, Elsevier Sc. Pub., Amsterdam, 1989

[16] M. De Leon and P.R. Rodrigues, Generalized Classical Mechanics and Field Theory, North-Hol. Math. St.,112, Elsevier Sc. Pub., Amsterdam, 1991.

[17] M. De Leon, D. Chinea and J.C. Marrero; The Constraint Algorithm for Time Dependent Lagrangians, Publ. Univ. La Laguna, Serie Informes, 32, 13-29,1991 